Forum Presèntation

\title{
AGE and Endothelial Cells
}

\author{
Sho-ichi Yamagishi, Hideto Yonekura, Yasuhiko Yamamoto, Hideki Fujimori, and \\ Hiroshi Yamamoto
}

Department of Biochemistry, Kanazawa University School of Medicine, Kanazawa, Japan.

\begin{abstract}
Advanced glycation endproducts (AGE) are non-enzymatically glycated protein derivatives, which are formed acceleratedly under hyperglycemic conditions. Here we describe effects of AGE on the growth and function of endothelial cells (EC). When human umbilical vein EC are exposed to AGE, DNA synthesis as well as viable cell number is increased, while prostacyclin production is significantly inhibited. This is mainly mediated by the interaction between AGE and a cell surface receptor for AGE (RAGE). AGE also stimulate the growth and tube formation of microvascular EC, the key steps of angiogenesis, through an autocrine induction of vascular endothelial growth factor. Moreover, AGE increase plasminogen activatior inhibitor $\mathbf{1}$ production by microvascular $\mathrm{EC}$, thus attenuating the fibrinolytic activity. The results indicate that AGE-RAGE interactions may predispose to angiogenesis and thrombogenesis, thereby leading to the development and progression of diabetic angiopathies. J Atheroscler Thromb, $1998 ; 4: 141-143$.
\end{abstract}

Key words : Pericyte, RAGE, Vascular endothelial growth factor, Diabetes mellitus

\section{Background}

Microvessels are composed of two cell types, endothelial cells (EC) and pericytes. Using pericyte-EC coculture systems, we have shown previously that pericytes not only regulate the growth but also preserve the prostacyclin $\left(\mathrm{PGl}_{2}\right)$-producing ability and protect against lipid peroxide-induced injury of EC, playing an important role in the maintenance of microvascular homeostasis (Fig. 1) (1, 2). This has provided a basis for our understanding how EC derangements develop consequent to 'pericyte loss', the earliest histopathological hallmark in diabetic retinopathy $(3,4)$.

Glucose can react non-enzymatically with the amino groups of proteins to form reversible Schiff bases and then

Address for correspondence: Hiroshi Yamamoto, MD, Ph. D., Department of Biochemistry, Kanazawa University School of Medicine, 13-1 Takara-machi, Kanazawa 920-8640, Japan.

This article is presented in the Forum \#10 on Endothelial Cell Function and Genesis of Atherosclerotic Vascular Disease at the 29th Annual Meeting on the Japan Atherosclerosis Society in Tokyo, 1997.

Received March 20, 1998.
Amadori products. These early glycation products undergo further complex reactions to become irreversibly cross-linked, fluorescent derivatives termed advanced glycation endproducts (AGE) (5). Recently, we found that AGE inhibited the growth of pericytes through interactions with their receptor for AGE (RAGE) $(3,6)$, and proposed a novel mechanism for pericyte loss. The AGE-induced, RAGE-mediated decrease in pericyte num-

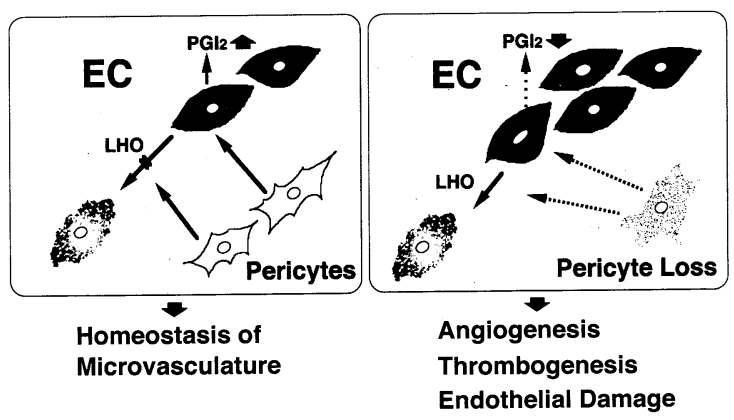

Fig. 1. Endothelial cell-pericyte interactions and possible sequelae of their disturbances. $E C$, endothelial cells. $\mathrm{PGI}_{2}$, prostacyclin. LHO, linoleate hydroperoxide. 
ber would then indirectly cause angiogenesis and thrombogenesis (1-3), participating in the development of diabetic vascular complications.

Our subsequent experiments revealed direct effects of AGE on EC. In this paper we describe how the growth and function of EC are affected by the senescent macroproteins formed acceleratedly in diabetes.

\section{Angiogenic Activity of AGE}

When human umbilical vein EC (HUVEC) and human skin microvascular EC were exposed to AGE-bovine serum albumin (BSA), their viable cell number as well as DNA synthesis was found to be significantly stimulated by AGE $(7,8)$. Neither non-glycated BSA nor Amadori compounds affected the EC growth, and the AGE effects were completely neutralized by antisera against either AGEBSA or AGE-RNase A (8), suggesting that common AGE structures may account for the growth promotion. This is the first demonstration that AGE could exert an angiogenic activity.

As shown previously $(3,6)$, HUVEC express the gene coding for RAGE, a cell surface receptor for AGE. So we next examined its functional role in the AGE action on HUVEC with an antisense strategy. When a phosphorothioate DNA complement of human RAGE mRNA was administered to the HUVEC culture, the angiogenic activity of AGE was found to be completely inhibited, while the sense control DNA induced no change (7). The results indicate that the AGE effect on EC growth is RAGEmediated.

AGE were also found to stimulate the tube formation of microvascular EC, the very cell type where angiogenesis takes place (8). We obtained evidence that it is vascular endothelial growth factor (VEGF) that mainly mediates the angiogenic activities of AGE (8). First, quantitative reverse transcription-polymerase chain reaction analysis revealed that AGE-BSA up-regulated the levels of mRNAs coding for the secretory forms of VEGF, while the levels of mRNAs for two VEGF receptors, kinase insert domain-containing receptor and fms-like tyrosine kinase 1, remained unchanged by the AGE treatment. Immunoprecipitation analysis revealed that AGE-BSA did increase de novo synthesis of VEGF proteins. Second, a monoclonal antibody against human VEGF (8) completely neutralized both the AGE-induced DNA synthesis and the tube formation of microvascular EC without discernible toxicity.

\section{Thrombogenicity of AGE}

AGE were found to inhibit HUVEC production of $\mathrm{PGI}_{2}$, the key substance endowing the endothelium with antithrombogenic activities (7). This inhibition was also proven to be RAGE-mediated, because RAGE antisense efficiently reversed the $A G E$-induced decrease in $\mathrm{PGI}_{2}$ production (7). Accordingly, AGE could predispose blood vessels to thrombogenesis by two modes of action; one, by acting on EC directly to impair their ability to produce $\mathrm{PGl}_{2}$, and the other, by decreasing viable pericyte number, which would result in the lack of support by this cell type to preserve EC functions including the synthesis of that antithrombogenic prostanoid $(1,3,7)$.

Furthermore, AGE were found to stimulate microvascular EC production of plasminogen activator inhibitor-1, a fast-acting serine protease inhibitor that attenuates fibrinolysis (9). The result suggests that AGE may contribute to platelet aggregation on one hand and promote fibrin stabilization on the other, which again predispose to thrombogenesis.

\section{Hypoxia and AGE}

Hypoxia is the principle cause of angiogenesis (10). We have shown previously that under hypoxic conditions VEGF productions are induced in both EC and pericytes and then promote the growth and tube formation of EC (11). Therefore, the AGE-induced thrombogenicity may give rise to focal ischemia and hypoxia, which in turn trigger autocrine and paracrine VEGF expressions, superdriving the microvessels toward angiogenesis, which may eventually lead to the full clinical expression of diabetic angiopathy.

\section{Conclusion}

AGE-RAGE interactions may thus play an active part in pericyte loss on one hand, and act on EC directly on the other, to predispose to angiogenesis and thrombogenesis, thereby leading to the development and exacerbation of diabetic micro- and macroangiopathies (Fig. 2). Based on this concept, procedures that can halt these events

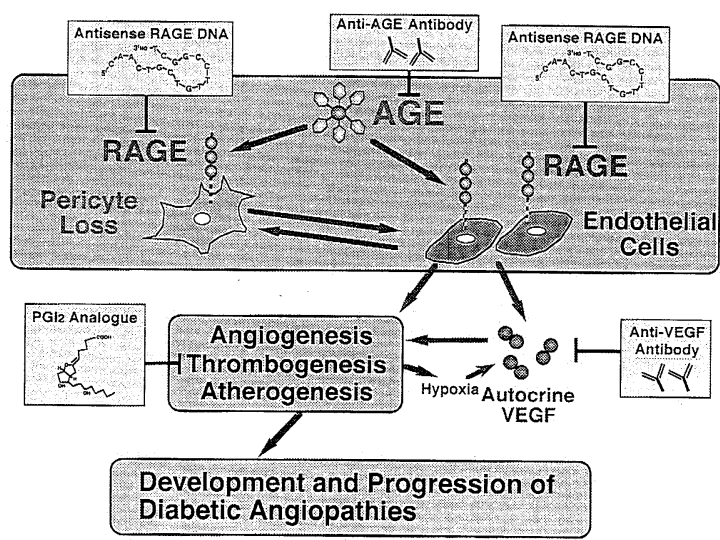

Fig. 2. Possible mechanisms for the development and progression of diabetic angiopathy and its prevention. AGE, advanced glycation endproducts. RAGE, a receptor for AGE. VEGF, vascular endothelial growth factor. $\mathrm{PGI}_{2}$, prostacyclin. 
would then theoretically help combat diabetic vascular complications; this would include inhibition of AGE formation, AGE apheresis by adsorption to immobilized antibodies, antisense RAGE DNA, prostacyclin analogues and anti-VEGF neutralizing antibodies (Fig. 2).

Acknowledgements: We thank Professor Yoshiya Hata, the President of the 29th Annual Meeting of Japan Atherosclerosis Society, for offering an opportunity to present this paper. This work has been supported in part by Grants-in-Aid for Scientific Research from the Ministry of Education, Science, Culture and Sports, Japan $(\mathrm{H}$. Ya, $H$. Yo and S. Y), from Chiyoda Mutual Life Foundation $(H$. Ya), from the Mochida Memorial Foundation for Medical and Pharmaceutical Research $(\mathrm{H}$. Yo), from the Japan Diabetes Foundation ( $\mathrm{H}$. Ya and S. Y), and from the Ichiro Kanehara Foundation (S. Y).

\section{References}

(1) Yamagishi S, Kobayashi K, and Yamamoto H: Vascular pericytes not only regulate growth, but also preserve prostacyclin-producing ability and protect against lipid peroxide-induced injury of co-cultured endothelial cells. Biochem Biophys Res Commun, 190: 418-425, 1993

(2) Yamagishi S, Hsu CC, Kobayashi K, and Yamamoto H: Endothelin 1 mediates endothelial cell-dependent proliferation of vascular peicytes. Biochem Biophys Res Commun, 191: 840-846, 1993

(3) Yamagishi S, Hsu CC, Taniguchi M, Harada S, Yamamoto $\mathrm{Y}$, Ohsawa K, Kobayashi K, and Yamamoto H: Receptor-mediated toxicity to pericytes of advanced glycosylation end products: A possible mechanism of pericyte loss in diabetic microangiopathy. Biochem Biophys Res Commun, 213: 681-687, 1995
(4) Kuwabara T and Kogan DG: Studies on the retinal vascular pattern. I. Normal Architecture. Arch Ophthalmol, 64 : 904-911, 1960

(5) Brownlee M, Cerami A, and Vlassara H: Advanced glycosylation end products in tissue and the biochemical basis of diabetic complications. $\mathrm{N}$ Engl $\mathrm{J}$ Med, 318: 1315-1321, 1988

(6) Neeper M, Schmidt AM, Brett J, Yan SD, Wang F, Pan YC, Ellison K, Stern D, and Shaw A : Cloning and expression of a cell surface receptor for advanced glycosylation end products of proteins. J Biol Chem, 267: 1499815004, 1992

(7) Yamagishi S, Yamamoto Y, Harada S, Hsu CC, and Yamamoto $\mathrm{H}$ : Advanced glycosylation end products stimulate the growth but inhibit the prostacyclin-producing ability of endothelial cells through interactions with their receptors. FEBS Lett, 384 : 103-106, 1996

(8) Yamagishi S, Yonekura H, Yamamoto Y, Katsuno K, Sato F, Mita I, Ooka H, Satozawa N, Kawakami T, Nomura M, and Yamamoto $\mathrm{H}$ : Advanced glycation endproductsdriven angiogenesis in vitro. Induction of the growth and tube formation of human microvascular endothelial cells through autocrine vascular endothelial growth factor. J Biol Chem, 272: 8723-8730, 1997

(9) Yamagishi S, Fujimori H, Yonekura H, Yamamoto Y, and Yamamoto $\mathrm{H}$ : Advanced glycation endproducts inhibit prostacyclin production and induce plasminogen activator inhibitor-1 in human microvascular endothelial cells. Diabetologia (in press)

(10) Yamamoto H: "Vascular VEGF" in hypoxia-induced proliferation of vascular cells. Microcirculation Annual 11: 11-12, 1995

(11) Nomura M, Yamagishi S, Harada S, Hayashi Y, Yamashima T, Yamashita J, and Yamamoto $\mathrm{H}$ : Possible participation of autocrine and paracrine vascular endothelial growth factors in hypoxia-induced proliferation of endothelial cells and pericytes. J Biol Chem, 270 : 2831628324, 1995 